\title{
Open Fracture of Pelvis
}

National Cancer Institute

\section{Source}

National Cancer Institute. Open Fracture of Pelvis. NCI Thesaurus. Code C35353.

A traumatic break in one or more of the bones comprising the bony structure of the pelvis that involves a break in the adjacent skin.. 